\title{
Coordinated Colorings and their Chromatic Groups
}

\author{
Agatha Kristel M. Abila ${ }^{1,2}$, Eduard C. Taganap ${ }^{3}$, Ma. Louise Antonette N. De las Peñas ${ }^{1}$
}

\author{
${ }^{1}$ Department of Mathematics, Ateneo de Manila University, Quezon City, Metro Manila 1108, Philippines, ${ }^{2}$ Southern Luzon State \\ University, Lucban, Quezon 4328, Philippines, ${ }^{3}$ Department of Mathematics and Physics, Central Luzon State University, Science \\ City of Munoz, Nueva Ecija 3120, Philippines \\ agathakristelabila@gmail.com
}

The study of chromatic symmetries to describe physical properties of crystals and mapping of individual orientations in twins has extended the field of mathematical crystallography. This paper discusses chromatic, partially chromatic or achromatic properties of symmetries or partial operations of a coordinated coloring of a symmetrical pattern, which has a potential to describe a crystalline structure. Here, we consider a symmetrical pattern $\mathcal{P}$ consisting of disjoint congruent copies of a symmetrical motif $\mathcal{M}$.

A coordinated coloring of $\mathcal{P}$ is a coloring that is perfect and transitive under the global symmetry group $G$ of $\mathcal{P}$, satisfying the condition that the coloring of $\mathcal{M}$ is also perfect and transitive under its symmetry group $K$ (a local symmetry group). This means that $G$ and $K$ consists of elements that effect a permutation of the colors of the coloring of $\mathcal{P}$ and $\mathcal{M}$ respectively. If the coloring of $\mathcal{P}$ or $\mathcal{M}$ has two (respectively more than two) colors, then $G$ or $K$ is called a dichromatic (respectively polychromatic) symmetry group.

A global symmetry, a local symmetry, or a partial operation (an isometry of the plane that sends one motif to another, which may not be a global or local symmetry of $\mathcal{P}$ ) of a coordinated coloring of $\mathcal{P}$ can be classified as either achromatic if it fixes all the colors; chromatic if it moves all the colors, and partially chromatic if it exchanges some colors, and fixes the rest [2].

As an example, consider the coordinated 4 - coloring of $\mathcal{P}$ given in Figure 1. Each region in every motif is assigned a color from the set $S=\{$ blue, yellow, red, green $\}$. Every element of $G=<20,0 ; m_{[10]} ; m_{[01]} ; z(1,0)>\cong \mathrm{p} 2 \mathrm{~mm}$ (a polychromatic symmetry group) permutes the colors in the 4 - coloring of $\mathcal{P}$. We also note that in this coloring, every element of $K=<4^{+} 0,0 ; m_{[10]}>\cong 4 \mathrm{~mm}$ permutes the colors assigned to the triangles in the motif $\mathcal{M}$. The dichromatic group of $\mathcal{M}$ is $4^{\prime} \mathrm{mm}^{\prime}$ : we have the $90^{\circ}$ rotation and two reflections that change colors, while two reflections fix colors. The dichromatic group of each of the other motifs is also $4^{\prime} \mathrm{mm}^{\prime}$.

The local chromatic symmetries of $M$ exchanging blue and red are $4^{\prime+}, 4^{\prime}, m_{[11]}^{\prime}$, and $m_{[11]}^{\prime}$. On the other hand, the local achromatic symmetries of $M$ fixing the colors blue and red are $2, m_{[10]}$, and $m_{[01]}$. The global symmetries in $\mathcal{P}$ can be characterized as: $z^{(4)}(1,0)$ (chromatic) sending blue to yellow, yellow to red, red to green, and green to blue; $2^{(2,2)} 0,0$, and $m_{[01]}^{(2,2)}$ (partially chromatic) exchanging yellow and green, and fixing red and blue; and $m_{[10]}$ (achromatic) fixing all the colors. The 4-coloring of the frieze pattern is described by the group $\left[p^{(4)} 2^{(2,2)} m^{(2,2)} m\right]^{(4)}$. The chromatic partial operations that map $M$ to $z M$ are also shown in Figure 1 . Sending blue to yellow as well as red to green are $z^{(2)}(1,0), 2^{(2)} \frac{1}{2}, 0, m_{[01]}^{(2)} \frac{1}{2}, 0$, and $g_{[01]}^{(2)}(1,0)$. Sending blue to green and red to yellow are $4^{(2)+} \frac{1}{2}, \frac{1}{2}, 4^{(2)-\frac{1}{2}, \frac{1}{2}}, g_{[11]}^{(2)}\left(\frac{1}{2}, \frac{1}{2}\right) \frac{1}{2}, 0$ and $g_{[11]}^{(2)}\left(\frac{1}{2}, \frac{1}{2}\right) \frac{1}{2}, 0$.

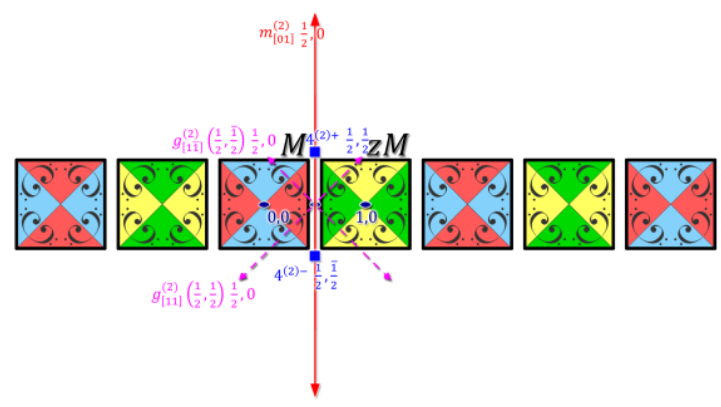

Figure 1. Partial operations relating $M$ to $z M$.

In this talk, examples of coordinated colorings of Frieze and Plane Crystallographic patterns will be presented.

[1] Sadanaga, R., Sawada, T., Ohsumi, K. \& Kamiya, K. (1980). J. Jpn.Assoc. Min. Petr. Econ. Geol. Spec. 2, $23-29$.

[2] Massimo Nespolo. The chromatic symmetry of twins and allotwins. Acta Crystallographica Section A Foundations and Advances, International Union of Crystallography, 2019, 75 (3), pp.551-573.

Keywords: local and global color symmetry, coordinated colorings, chromatic groups 